\title{
LES PRIÈRES DE L'AMBON DE LA LITURGIE DE SAINT JACQUES
}

Dans un premier article prospectif sur les "prières de l'ambon" (ỏ $\left.\pi \_\tau \alpha \dot{\alpha} \mu \beta \omega \nu \circ \varsigma\right)$ grecques et les prières de renvoi (ganTeveba) géorgiennes, ${ }^{1} \mathrm{j}^{\prime}$ ai entrepris un premier tour de la question et risqué quelques hypothèses sur la datation de la Vorlage grecque de la collection géorgienne ; j'y reviendrai à propos du caractère « origéniste » d'une autre prière que celle examinée dans ce premier article ; mais je voudrais ici surtout $1^{\circ}$ ) expliquer l'évolution de la prière de renvoi dans la Liturgie de saint Jacques, ${ }^{2} 2^{\circ}$ ) traduire les incipit des «prières de renvoi » du $\sin$. géorgien $12,{ }^{3} 3^{\circ}$ ) donner la liste des prières grecques correspondantes. ${ }^{4}$

\section{A. Une rubrique de la Liturgie de saint Jacques}

Alors que la description de la fin de la messe par Égérie ${ }^{5}$ se contente d'une allusion à une bénédiction de l'évêque $(25,3)$, deux rubriques de

(1) S. Verhelst, Prières géorgiennes de renvoi et prières grecques de l'ambon. Premières comparaisons, BBGG 2009, à paraître (plus loin = VERHELST, $B B G G)$.

(2) Comme le rappelle S. PARenti, Testimoni sconosciuti di preghiere dell'ambone, OCP 62 (1996) 197-205 (= PARenti, OCP 1996), v. p. 204, Baumstark est le premier à avoir noté l'origine hagiopolite de la prière de l'ambon ; R. F. TAFt, Toward the Origins of the Opisthambonos Prayer of the Byzantine Eucharistic Liturgies, $1^{\mathrm{e}}$ part., OCP 72 (2006) 5-39 ; $2^{\mathrm{e}}$ part., OCP 72 (2006) 305333 (= TAFT, OCP 2006), p. 311-331 a aussi développé ce point de vue.

(3) Et occasionnellement du sin. gé. 54 ; sur ces deux manuscrits, v. la brève description de Xevsuriani dans R. Gvaramia, E. Metreveli, C. Çankievi, L. Xevsuriani, L. Djghamaia, Description des manuscripts géorgiens. Collection du Sinaï, t. III (Tbilisi, 1987) (en géo.) (= Gvaramia et al., 1987) 36-38 et 60.

(4) Je renonce par contre à publier mon répertoire des prières de l'ambon, puisque R. F. Taft a annoncé la publication du sien (R. F. TAFT, Proper Slavonic Opisthambonos Prayers, Studi sull'Oriente Cristiano 10/2 (2006) 133-149 (= TAFT, SOC 2006), p. 136); je le compléterai si nécessaire sur le site internet sur la liturgie de Jérusalem que j'espère pouvoir créer prochainement (comportant le nom scholasate dans son titre).

(5) P. Maraval, Égérie. Journal de voyage (Itinéraire) (Paris, 1982) (SC, 296). 
la Liturgie de saint Jacques certainement très anciennes rappellent la fin de la Liturgie des Constitutions apostoliques (VIII, 15) : une prière d'action de grâces pour l'eucharisite suivie d'une prière sur les fidèles inclinés, soit, dans ma numérotation des rubriques de Saint-Jacques : ${ }^{6}$

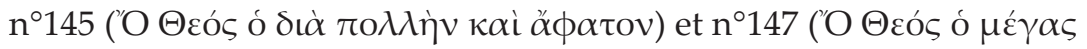

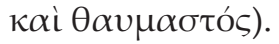

Il est possible que cette dernière prière se disait face à l'assemblée ; d'abord parce qu'Égérie dit que l'évêque "bénit » les fidèles, ce qui se fait mieux avec le geste des mains élevées face au peuple $;^{7}$ ensuite parce que ce geste (et cette prière) rappelle étroitement un geste (et une prière) similaire à la fin des offices de la liturgie juive, accompagnant la birkat kohanîm, c'est-à-dire la lecture solennelle de Nb. 6, 2426 par les kohanîm (à quoi font précisément allusion les Constitutions apostoliques, II, 57, 19) ; finalement parce que certains manuscrits ont conservé la mention du prêtre se tournant vers les fidèles à la fin de la Liturgie, même si ce n'est plus à l'occasion du n 147 (mais du n ${ }^{\circ} 155$, v. ci-dessous).

Des rubriques se sont en effet ajoutées à la fin de la Liturgie (comme d'ailleurs au début), suivant cette loi de redondance aux extrêmes qui caractérise le développement de l'histoire de la liturgie. ${ }^{9}$ Les deux

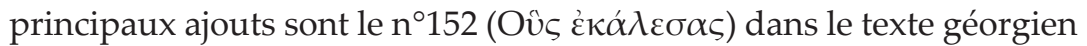

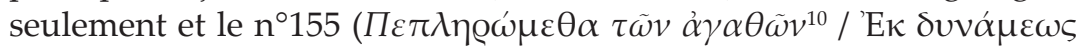

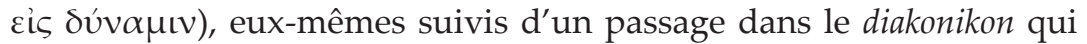
a donné lieu à l'insertion de nouvelles rubriques. Il s'agit non seule-

(6) Voir Liturgia ibero-graeca, à paraître dans la collection Jerusalemer Theologisches Forum (Münster).

(7) En toute rigueur, le texte d'Égérie, 24, 6-7 (ou celui des Constitutions apostoliques, VIII 37, 4-6; 39, 1-4; 41, 6-8) dit que la bénédiction épiscopale se donne après que les fidèles ont incliné la tête; il est donc possible qu'il y ait eu d'abord une prière (non mentionnée) face aux fidèles inclinés, sur eux, et une seconde, orientée vers le fond de l'église, correspondant à la "bénédiction" (ici le $\mathrm{n}^{\circ} 147$ ).

(8) Sur la birkat kohanîm, v. I. Elbogen, Jewish Liturgy. A Comprehensive History (1éd. all. 1913, 2e éd. héb. 1972, Jérusalem-New York, 1993) 54-57 $(=\S 9 \mathrm{a})$.

(9) Ce qu'a montré en particulier J. Heinemann pour la tradition juive, Prayer in the Talmud. Forms and Patterns (St. Jud. IX) (1 $1^{\mathrm{e}}$ éd. héb. 1964, BerlinNew York, 1977) 163-164, 266-267.

(10) En italiques la rétroversion grecque du texte géorgien non attesté en grec. 
ment de prières ${ }^{11}$ mais d'une synaptie diaconale $\left(=n^{\circ} 153\right)$. Celle-ci, tout comme les prières de renvoi géorgiennes, est l'objet d'une série de variantes regroupées dans une collection de l'euchologe de Jérusalem conservé en géorgien, une collection dont je ne reparlerai pas ici bien qu'elle se trouve dans les mêmes manuscrits que ceux qui ont conservé la collection de prières de renvoi. ${ }^{12}$

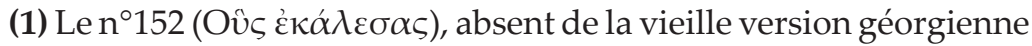
(tbilisi A 86), bien qu'il n'ait pas été conservé dans les manuscrits grecs de Saint-Jacques, est toutefois attesté en grec (notamment), et cela justement avec le titre "prière de l'ambon", dans l'euchologe sin. grec $961 .{ }^{13}$ Les trois manuscrits géorgiens sinaïtiques où elle est transcrite ne lui ont pas donné d'appellation particulière, mais elle fait partie de la collection des prières de renvoi des $\sin .12$ et 54 (v. plus loin $n^{\circ} 62$ ).

(2) Le ${ }^{\circ} 155(\Pi \varepsilon \pi \lambda \eta \varrho \omega ́ \mu \varepsilon \theta \alpha \tau \tilde{\omega} v \dot{\alpha} \gamma \alpha \theta \tilde{\omega} v$ - texte géorgien / 'Eк

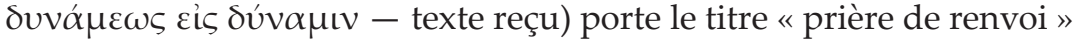
dans deux manuscrits géorgiens et, quant à lui, s'est transmis dans Saint-Jacques grec bien que certains manuscrits, en modifiant son incipit, en font une prière « de l'autel au diakonikon » (mss IE) ${ }_{1}^{14}$ ou, plus souvent, une prière à dire dans le diakonikon même (mss MHLKA). ${ }^{15}$

Les $n^{\circ} 152$ et 155 sont deux variantes d'une même rubrique. ${ }^{16}$ Un seul manuscrit de Saint-Jacques, celui de Jean Zosime édité par Tarxnišvili (G), possède les deux numéros et il n'y a pas lieu de distinguer une rubrique " prière de l'ambon » qui serait le $\mathrm{n}^{\circ} 152 \mathrm{~d}^{\prime}$ une rubrique

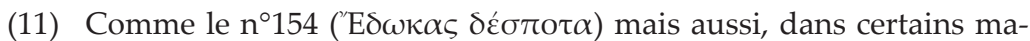
nuscrits, le ${ }^{\circ} 155$ lui-même ; rubrique connue aussi sous le nom "prière du skeuophylakion».

(12) Aux trois manuscrits contenant la collection des prières de renvoi ( $\sin$. 12 et 54, N/Sin 26) s'ajoute, pour la collection de synapties diaconales dans le diakonikon, le N/Sin 53.

(13) А. ЈАСов, Les prières de l'ambon du Barb. gr. 336 et du Vat. gr. 1833, Bulletin de l'Institut historique belge de Rome 37 (1966) 17-51 (= JАсов, BIHBR $1966=$ JАСОВ, I) ; IDEM, Une prière du skeuophylakion de la Liturgie de saint Jacques et ses parallèles byzantins, ibid., 53-80, v. p. 57.

(14) PO 26, p. 244, ad 1. 20 (skeuophylakion E).

(15) Sigles de l'édition de Mercier (C. Mercier, La Liturgie de saint Jacques: édition critique du texte grec avec traduction latine, PO 26 (1946) 115256) auxquels s'ajoutent le koutloumoussiou 194, XIV e s. (K) et le sinaïticus grec 1039, XIII' s. (L).

(16) Comme on peut le conclure déjà de l'article de ЈАСов, I, p. 57 et 75. 
« prière de renvoi » final qui serait le $n^{\circ} 155$. En réalité le titre « prière de l'ambon » n'apparaît pas en géorgien. Ce titre vient très probablement de Constantinople, où l'ambon, à Sainte-Sophie en particulier, avait des dimensions considérables ${ }^{17}$ alors que ce type d'ambon n'est guère attesté en Palestine. ${ }^{18}$

Pourquoi s'est introduite cette rubrique en plus de la prière de renvoi habituelle, à savoir le $\mathrm{n}^{\circ} 147$ (qui est en réalité une prière d'inclination) ? Le $\mathrm{n}^{\circ} 147$ devait se dire à l'origine face au peuple et sans doute en avant du sanctuaire, le plus près possible des fidèles attendant du célébrant le geste de ses mains sur leur tête inclinée ; la prière de l'ambon a dû s'introduire au moment où le prêtre se retournant vers l'autel et se trouvant pratiquement hors du sanctuaire, est sur le point d'y retourner. La rubrique de IE pour le ${ }^{\circ} 155$ (« de l'autel au diakonikon ») y fait précisément allusion (v. note 14).

Est-ce à dire que toutes les prières de l'ambon soient palestiniennes ? Le plus ancien manuscrit grec, le barb. grec 336, attribue l'une d'entre elles à Germain, patriarche de Constantinople en 715-730. Il n'y a pas de raison de mettre en doute une datation au début du VIII s. de la pénétration de la rubrique palestinienne à Constantinople. Jérusalem étant désormais sous souveraineté arabe, la capitale byzantine se devait de jouer un rôle dans la conservation des traditions palestiniennes. Mais le fait que plusieurs manuscrits grecs de prières ỏ $\pi \iota \sigma \tau \alpha ́ \alpha \beta \omega \nu o \varsigma$ soient des manuscrits copiés en Italie méridionale, montre que, si la rubrique a pénétré dès cette époque à Constantinople, où elle aurait reçu son nom, ${ }^{19}$ c'est en Italie méridionale qu'elle s'est le mieux transmise. Reste à savoir si les prières grecques conservées sont anciennes (pré-arabes) ou au contraire le résultat d'une réforme post-iconoclaste ${ }^{20}$. Des éléments de réponse se trouvent dans les prières géorgiennes.

(17) А. ЈАСов, Où était récitée la prière de l'ambon, Byzantion, 51 (1981) 306-315 (= ЈАСОВ, Byz 1981), p. 311, 313-314 ; ТАFт, ОСP 2006, p. 14-17 ; v. cependant la remarque plus loin (note 18).

(18) Le stavrou 43 montre qu'une telle structure existait dans l'église de l'Anastasis, mais la question est de savoir si elle y était avant sa destruction en 1009 (ainsi TAFT, OCP 2006, p. 318), ou dans le cadre de sa reconstruction au milieu du XI ${ }^{\mathrm{e}}$ siècle.

(19) À vrai dire, puisque les ambons sont assez communs dans les églises d'Italie, on pourrait se demander si le titre «prière de l'ambon » ne vient pas d'Italie.

(20) Selon une thèse de Passarelli, démontée par ЈАСов, Byzantion (1981), p. 307-311. 


\section{B. Liste des prières de renvoi de la Liturgie de saint Jacques}

Sur base de ces clarifications, continuons notre énumération des « prières de l'ambon / de renvoi » d'après la tradition manuscrite de la Liturgie de saint Jacques :

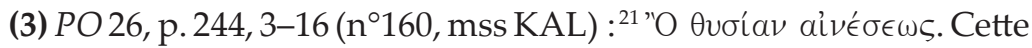

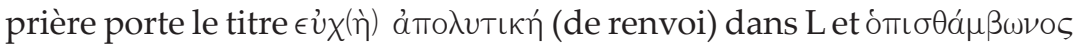
єủxฑ́ dans AK.

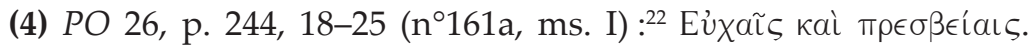

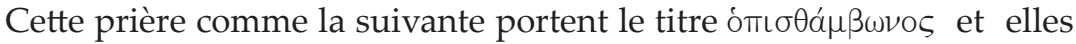
se disent toutes deux, au choix, avant le passage dans le diakonikon, indiqué par la rubrique du $\mathrm{n}^{\circ} 155$ qui les suit. ${ }^{23}$ Son contenu est en fait une prière diaconale, reliquat grec de la rubrique diaconale se disant dans le diakonikon ( $\mathrm{n}^{\circ} 153$ de $\mathrm{SJ}$, v. ci-dessus note 12$)$.

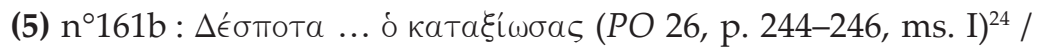

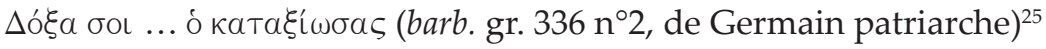

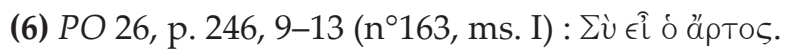

La rubrique se dit après le ${ }^{\circ} 155$ comme « autre prière ». Bien que le $\mathrm{n}^{\circ} 155$ (ci-dessus n`2) ne soit pas une «prière de l'ambon » dans le vat. grec 1970 (ms. I), elle en tient lieu (« de l'autel au diakonikon ») et mérite à ce titre de figurer dans le catalogue, même si, dans certains manuscrits, d'autres prières prennent aussi la place d'une prière de l'ambon.

(21) Aussi dans JАСОВ, I, p. 23-24; 40-41; IDEM, Nouveaux documents italo-grecs pour servir à l'histoire du texte des prières de l'ambon, Bulletin de l'Institut historique belge de Rome 38 (1967) 109-144 (= ЈАСОВ, II), p. 112 ; IDEM, Les prières de l'ambon du Leningr. gr. 226, ibid. 42 (1972), 109-139 (= ЈАСов, III), p. 126-127.

(22) Rubrique similaire dans le seul manuscrit géorgien G (de Jean Zosime); M. TaRChNišvili, Liturgix Iberice antiquores (Louvain, 1950) (CSCO, $122,123)$ (= TARCHNIšvili, 1950), p. 24 (trad.), n³5.

(23) Cette disposition des rubriques n'est pas indiquée clairement par Mercier (malgré PO 26, p. 158); elle se voit seulement dans l'édition de C. A. SwaInson, The Greek Liturgies, chiefly from original authorities with an appendix containing the Coptic ordinary canon of the mass from two manuscripts in the British Museum (Londres, 1884 ; réimpr. Hildesheim, 1971).

(24) Aussi dans ЈАсов, III, p. 123 et А. ДмитриЕвский, Описание литургических рукописей, хранящихся в библиотеках Православного Востока. Вып. II: Euchologia (Киев, 1901 ; réimp. Hildesheim, 1965) (= DмiтrievsкiJ, II), p. 43.

(25) ЈАСов, I, p. 21-22. 
C'est le cas du n ${ }^{\circ} 154$ dans $\mathrm{H}^{26}$ Celle-ci est cependant à l'origine, comme le prouve le texte géorgien, non pas une «prière après la communion » $(\mathrm{H})$ mais une prière à dire dans le diakonikon. ${ }^{27}$

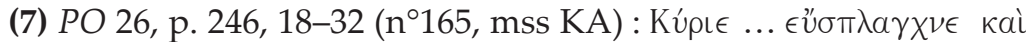
$\pi \circ \lambda v \dot{\epsilon} \lambda \epsilon \in{ }^{28}$

Prière pour le carême; $\mu \in T a \lambda \eta \dot{\psi} \psi \epsilon \omega \varsigma$ dans le titre selon l'édition de

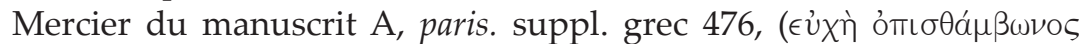

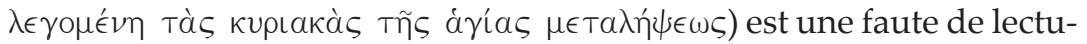
re de « $\mathrm{M}$ », « quarantaine ». Dans $\mathrm{K}$, elle est déplacée après la Liturgie de saint Pierre. On la trouve aussi dans le crypt. grec $\Gamma \beta \mathrm{X}$, avec dans le

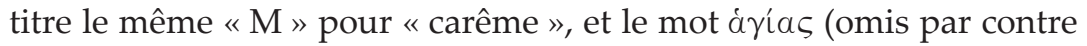
par K).

Cette prière a la particularité d'énumérer des jeûnes bibliques: celui des Ninivites (Jon. 3, 5-10), de Daniel dans la fosse aux lions (Dn. 6), ${ }^{29}$ de Moïse (Dt. 9, 9), d'Élie lors de son enlèvement par le char de feu (IV Rg. 2, rapproché de III Rg. 19, 8), du publicain (Lc 18), ${ }^{30}$ de la pécheresse de Lc 7, 36-50, ${ }^{31}$ auxquels le manuscrit crypt. grec $\Gamma \beta \mathrm{X}$ ajoute Pierre dans l'épisode de Corneille, où il n'est pas question de jeûne (mais d'abstinence, v. Ac. 10, 14). La liste est ainsi portée à sept exemples de jeûne, et il y a tout lieu de penser que c'était son intention primitive, comme dans les homélies sur le jeûne. ${ }^{32}$

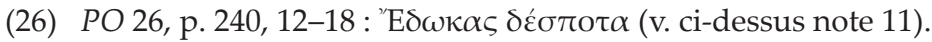

(27) Ceci rend caduques certaines observations de TAFT, OCP 2006, p. 322.

(28) Aussi dans JАСов, I, p. 24-25.

(29) Comme dans l'homélie sur le jeûne de Jean de Bolnisi, v. S. Verhelst, Jean évêque de Bolnisi en Ibérie caucasienne au début du IXe siècle. Homélies des dimanches de carême suivant la tradition de Jérusalem et autres homélies traduites et commentées, précédées d'une introduction sur l'auteur et le sujet. Texte géorgien de Sophio Sardjueladze et al., à paraitre dans la collection des SC (Paris) (= VERHELST, SC), ch. 1 (avec le commentaire dans l'introduction); le jeûne de Daniel n'est en fait mentionné qu'en Dn. 10, 3.

(30) Comme dans l'homélie sur le pharisien et le publicain de Jean de Bolnisi, où l'on trouve d'ailleurs aussi une énumération de jeûnes bibliques, $\mathrm{v}$. VERHELST, SC, ch. 7.

(31) Péricopes du huitiême dimanche avant Pâques attestée par les sources géorgiennes (notamment la troisième homélie de Jean de Bolnisi) mais omise dans le lectionnaire.

(32) Voir mon commentaire cité note 29. 
Alors que le texte de K ne contient aucune variante substantielle par rapport au texte édité, le crypt. grec $\Gamma \beta \mathrm{X}$ a les variantes suivantes :

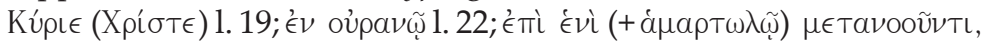

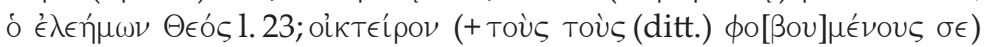

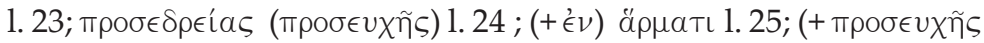

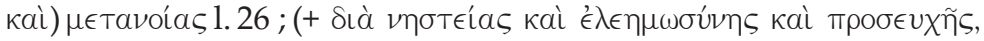

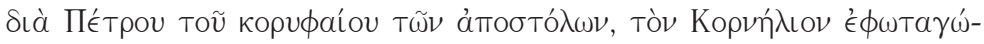

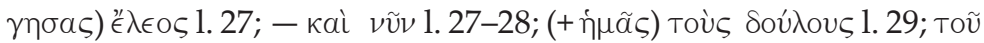

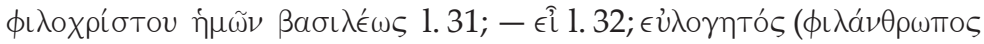

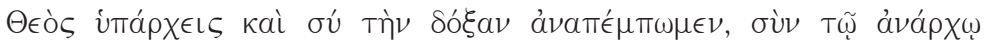

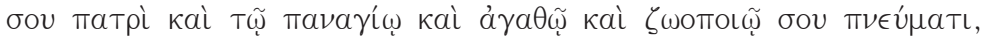

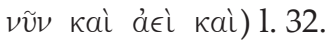

Dans le manuscrit $\mathrm{H}$ (vat. grec 2282), trois « prières de renvoi » plus l'incipit d'une quatrième (éditées par Cozza-Luzzi) ${ }^{33}$ s'ajoutent comme des variantes à la « prière finale » $\mathrm{n}^{\circ} 158$ (PO 26, p. 242, 2-6), après laquelle elles se trouvent. La rubrique du $\mathrm{n}^{\circ} 155$ indique pourtant que l'on est déjà dans le diakonikon, signe probable que la rubrique n'était plus conservée que comme un reliquat de la rubrique primitive, tombée en désuétude à l'endroit (Damas) et à la date de la rédaction de ce manuscrit (au XI ${ }^{e}$ s.). ${ }^{34}$ La première ne semble pas attestée ailleurs, les deux suivantes se trouvent dans les manuscrits édités par Minisci pour les dimanches ordinaires. Quant à l'incipit de la quatrième, on le retrouve dans une prière de l'ambon pour la fête de l'Exaltation de la Croix du vat. grec 2032, d'où elle a été éditée par Jacob.

(8) Ti $\dot{\alpha} \nu \tau \alpha \mu \imath \psi \omega ́ \mu \epsilon \theta a$. Que pouvons-nous te répondre, ô Verbe très souverain du Père à qui appartient tout commencement, que pouvonsnous t'apporter en échange, à toi le donateur de tout culte

(9) Mєбíta $\Theta \epsilon$ ṽ. Médiateur de Dieu et des hommes, Christ notre Dieu, Artisan, Créateur et Rédempteur de notre nature (Minisci, IV, p. 3-4)

Cette prière comporte à la fin une demande pour la victoire du roi contre les ennemis.

(33) G. Cozza-Luzzi, De Vetusta Liturgia Antiochena ex rotulo membranaceo uncialibus characteribus descripto, (Rome, 1905) (Novae Patrum Bibliothecæ ab Ang. Car. Maio collectæ, t. 10, pars secunda) (=CozzA-Luzzi, 1905), p. 105-110, la troisième est adressée au Père.

(34) Et non au IX comme le voulait Mercier, PO 26, p. 134, v. S. Verhelst, Les traditions judéo-chrétiennes dans la liturgie de Jérusalem, spécialement la Liturgie de saint Jacques frère de Dieu (Leuven, 2003) (Textes et études liturgiques. Studies in Liturgy, 18) (= Verhelst, 2003), p. 14. 
(10) ※Афатоৎ. Ton amour pour nous est ineffable, immense est l'océan de ta bonté (Minisci, IV, p. 5) ${ }^{35}$

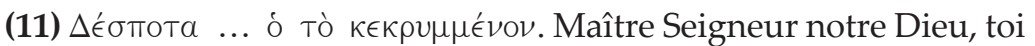
qui manifestes à la fin, par la croix et la résurrection, le mystère caché depuis les générations et depuis les siècles, afin de forcer celui qui possède la puissance de la mort, c'est-à-dire le diable, et de libérer ta créature de son emprise (JАСОВ, II, p. 123-124)

Les nouveaux manuscrits grecs sinaïtiques de la Liturgie de saint Jacques conservent peut-être d'autres « prières de renvoi » ou « prières de l'ambon ${ }^{36}{ }^{36}$ ainsi que les versions araméenne (du nouveau fonds sinaïtique, inédite), arabe ( $\sin .247$, inédite) et slavonne (éd. Syrku). ${ }^{37}$

On peut terminer ce tour d'horizon des témoins directs de la messe de Jérusalem en grec par le rubricaire de la semaine sainte stavrou 43, qui contient plusieurs prières de renvoi ou d'ambon - les deux titres apparaissent - pour les jours de la Grande Semaine. ${ }^{38}$ Seule la seconde est originale, les autres étant reproduites aussi ailleurs.

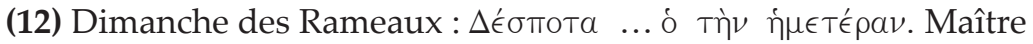
Christ notre Dieu, toi qui (...) as saisi l'axe (mółov) du ciel en montant et t'installant sur les Chérubins, qui aujourd'hui pour tout axe as accepté d'être mis et installé sur l'ânon $(\pi \omega \dot{\lambda} \lambda \omega)$ d'un âne de bât. ${ }^{39}$

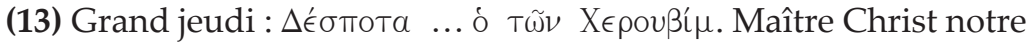
Dieu, toi qui chevauches les Chérubins et tiens les rênes des Séraphins, démiurge et maître du monde entier.

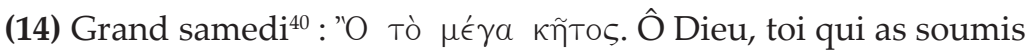
le grand monstre marin et qui dans le cœur de la terre as affronté le dragon révolté, le grand esprit des Assyriens, et qui lui as donné des ordres pour te moquer (dans le texte de Minisci : Ô Dieu, toi qui vas

(35) Aussi Јасов, I, p. 44-45; DмitrievskiJ, II, p. 43.

(36) Il s'agit des manuscrits suivants: M 151, X 156, E 23, E 79 (?) et $\times 277$.

(37) P. Syrku, De historia correctionis librorum in Bulgaria sæculo XIV, t. I (Saint-Pétersbourg, 1890) 179-218 cité par Goussen dans OC (1913), p. 2-3, Hanssens dans OCP 4, p. 250-251 ou encore Mercier, dans PO 26, p. 129.

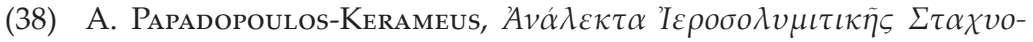

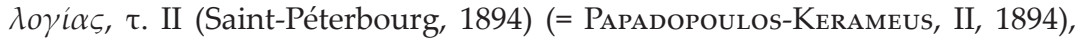
respectivement p. 27-28; 107-108; 188-189; 202-203.

(39) Aussi dans Јасов, II, p. 127-128.

(40) Après la $2^{\mathrm{e}}$ Liturgie mais avant l'orthros 
soumettre le grand monstre marin et qui durant cette nuit relèves de l'Hadès le grand berger des brebis spirituelles). ${ }^{41}$

(15) Pâques: $\Lambda \alpha ́ \mu \pi \rho \alpha$ i் $\mu \tilde{\nu}$. Brillante et salvatrice s'est levée pour nous aujourd'hui, frères, la résurrection de Notre-Seigneur JésusChrist, et c'est pourquoi le temple du Seigneur est orné d'hommes divers; voici en effet beaucoup de frères se sont rencontrés rendus joyeux par le jeûne. ${ }^{42}$

\section{Traductions des incipit des prières géorgiennes de renvoi et indication des parallèles}

\section{Références aux éditions des prières}

CL = CozzA-LuzzI, 1905, p. 105-110 ; DM = DMitrievsKIJ, II, 1901 ; J I II III = JАСов, BIHBR 1966, 1967, 1972 ; Ko = Kovaliv, 1960 ;3 M II III IV = Minisci, BBGG 1949-1951; PK = PAPAdopoulos-KerameUs, II, $1894 ;$ PAR = PArenti, OCP $1996 ;$ PAs = PAssarelli, $1982 ; 44$ Ver = VerHELST, BBGG. Les chiffres à la suite des références renvoient toujours aux pages.

\section{(16) 1. Annonciation (35rv)}

Pour la même circonstance : n91 (M III 7 ; J III 129), 156 (J II 136137), 161 (J II 112-113)

$=\sin .54, \mathrm{n}^{\circ} 1$ (f. $29 \mathrm{rv}$ )

$\approx \mathrm{n}^{\circ} 91$

Prière de renvoi pour l'Annonciation. Toi qui as envoyé un ange de paix à la très-sainte Vierge et Génitrice de Dieu Marie ${ }^{45}$

(17) 2. Dimanche avant Noël (35v-37r)

Pour la même circonstance : n75 (M II 74), 143 (M III 192-193)

Prière de renvoi un dimanche avant la nativité du Christ. Tu es glorifié, ô Seigneur, et rien n'est accessible à ta richesse, tu es terrible,

(41) T. Minisci, Le preghiere opistamvoni dei codici criptensi, BBGG 2 (1948) 65-75, 117-126 ; 3 (1949) 3-10, 61-66, 121-132 ; 4 (1950) 3-14 (= MiniscI, II, III, IV), III, p. 65.

(42) Aussi dans Minisci, III, p. 121-123 (surtout d'après le ms. i) ; JАСOB, II, p. 115 et III, p. 121.

(43) P. Kovaliv, Prayer Book. A Monument of the XIV Century (New York, 1960) (Scientific Theological Institute of the Ukranian Orthodox Church of the U.S.A.) (en ukrainien).

(44) G. Passarelli, L'eucologio cryptense T. $\beta$. VII (sec. X) (Thessalonique,

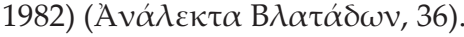

(45) Suite de la traduction dans BBGG. 
ô notre Dieu, et rien n'est l'égal de ta sagesse, ô ineffable. Merveilleuses sont tes œuvres, ô notre sauveur, et il n'est pas possible de scruter l'immense miséricorde de tes bienfaits qui, dans ta merveille inépuisable, est apparue à la race des hommes.

(18) 3. Veille de Noël (37r-38v)

Pour la même circonstance : $\mathrm{n}^{\circ} 76$ (M II 75)

Prière de renvoi le jour avant la nativité du Christ. Dieu sans commencement qui as envoyé ton Fils-Unique et Verbe au monde, Dieu de Dieu, lumière de lumière, coexistant et coéternel du Saint-Esprit incompréhensible et plus éminent que (toute) grandeur ; dans la pauvreté de ta divinité et la richesse de l'infirmité humaine, il est descendu du ciel comme une pluie de douceur sur une toison, et il a fait sa demeure dans le sein d'une vierge, comme dans un temple de sainteté, lui saint des saints

(19) 4. Noël, 25/12 (38v-39r)

Pour la même circonstance : n77 (M II 117 ; J III 128-129), 138 (J I 46 ; III 123), 144 (M III 189), 163 (J II 116)

$=\sin .54, \mathrm{n}^{\circ} 2($ f. $29 \mathrm{v}-30 \mathrm{r})$

$\approx \mathrm{n}^{\circ} 163$.

Renvoi pour la nativité du Christ, à l'eucharistie. Toi qui es grand dans le conseil de tes saints ô Dieu ${ }^{46}$

(20) 5. David-Jacques, 26/12 (39r-40v)

De David-Jacques. Toi qui fus Verbe avant les siècles et Dieu de commun conseil que le Père, et qui à l'achèvement des temps, Verbe, pris chair de la très sainte Vierge

(21) 6. Stéphane, 27/12 (40v-41v)

Pour la même circonstance : $\mathrm{n}^{\circ} 78$ (M II 118)

Renvoi de saint Stéphane. À chaque époque, en vision, ${ }^{47}$ des bouches te glorifieront, (comme celle) de Moïse, premier prophète et premier légiste en Israël - mais lui, comme serviteur de la loi, s'est caché dans le creux d'un rocher. Stéphane quant à luii ${ }^{48}$ témoigna comme diacre de la grâce après ta crucifixion et résurrection et dit : celui sur qui vous avez voulu mettre la main et que vous avez cloué sur le bois, lui, est ressuscité des morts. Et moi je témoigne et je (le) vois siégeant sur un trône à la droite du Père

(46) Suite de la traduction dans BBGG.

(47) + des deux ms.

(48) + lorsqu'il ms. 


\section{(22) 7. Jean et Jacques, 28/12 (41v-43r)}

Renvoi des apôtres Jean (et Jacques). Depuis le commencement le Verbe était, et Dieu était le Verbe. ${ }^{49}$ C'était depuis le commencement toi, avec Dieu et avec le Père en même temps que ton Esprit sans commencement et de la même existence, glorifié. Toutes les choses ellesmêmes de lui ont été faites, ${ }^{50}$ éternelles, (lui) qui est l'éclat de (ta) gloire et l'image de ta puissance ${ }^{51}$

(23) 8. Commun des saints, 22/01 (43r-v)

Pour la même circonstance : ${ }^{\circ} 110$ (M III 194), 129 (J I 36), 130 (J I 36), 138 (J I 46 ; III 123), 153 (M III 193-194), 169 (M III 194)

$\approx$ (incipit) $\mathrm{n}^{\circ} 153$

$=\mathrm{n}^{\circ} 57$

Renvoi des saints. Dieu saint qui reposes parmi les saints, toi qui as institué la mémoire des saints martyrs, qui ont donné leur corps pour un jugement

\section{(24) 9. dimanche avant Épiphanie (43v-45r)}

Renvoi un dimanche avant l'Épiphanie. Lumière véritable, Christ notre Dieu, Verbe du Père et du Saint-Esprit, de même existence et de même souveraineté, toi qui étais premier du sein éternel du Père, indiciblement et insaisissablement Dieu engendré de Dieu, lumière émise d'une lumière, vie issue d'une vie. Nous te rendons grâces, tout-puissant et dispensateur de tout bien

(25) 10. Épiphanie, 6/01 (45r-46r)

Pour la même circonstance : nº1 (M II 121-122 ; J II 117, 118), 115 (M II 121), 146 (M III 189), 171 (PAR 198-199)

$=\sin .54, \mathrm{n}^{\circ} 4(31 \mathrm{v}-32 \mathrm{v})$

Renvoi pour l'Épiphanie, à la messe. Tu t'es manifesté au monde dans la chair, comme tu es un Dieu, premier de toutes les créatures, ${ }^{52}$ avec le Père incorruptible et ton Esprit existant, véritable. Ô toi qui créas le monde du non-existant - et tu as conduit toutes les choses faites à l'existence - , fais visiter la lumière de la vie et la résurrection dans l'obscurité et les ombres de la mort ; fais descendre dans la rivière le créateur des mers et sur nous la lumière, toi qui créas notre race

(49) Jn 1,1

(50) Col. 1, 16a

(51) Voir le commentaire dans BBGG.

(52) Col. 1, 15 
(26) 11. dimanche après Épiphanie (46v-47v)

Renvoi pour le dimanche après l'Épiphanie. Toi que louent des troupes nombreuses d'anges, ô roi des cieux, et qui es ouvertement glorifié par des puissances célestes, nous bénissons nous aussi, tes serviteurs humbles et indignes, ta bonté ineffable

(27) 12. Commun des saints pères, 28/01 (47v-49r)

Pour la même circonstance (?), commun des pieux, etc. : n¹23 (M III 132), 154 (J II 129 ; M IV 3)

Renvoi pour les saints pères. Toi qui as appelé les élus par une voix bienheureuse

Remarque: La prière cite Élie et Jean-Baptiste, modèles des saints ascètes.

(28) 13. Hypapante (49r-50v)

Pour la même circonstance : n 82 (M II 122 ; J III 115), 147 (J II 125)

$=\sin .54, \mathrm{n}^{\circ} 5(32 \mathrm{v}-33 \mathrm{v})^{53}$

Renvoi pour l'Hypapante. Lumière véritable, coexistant avec le Père et coéternelle avec l'Esprit saint, toi qui avant les siècles es né du Père, qui inclinas les cieux et descendis sur la terre dans le sein d'une vierge et y habitas. Dans ta grande magnificence ${ }^{54}$ tu t'appauvris toimême (...). Toi qui ornas la terre de plante, de fleur de diverses espèces, tu fis resplendir une vierge plus qu'un paradis. Et dans le temple le vieillard Siméon te reçut. ${ }^{55}$ Par (son) annonce de ta divinité dans les cieux sur les chérubins avec le Père tu étais glorifié et dans le temple assis dans les bras des prophètes comme un autre Dieu et un autre homme tu étais présenté au peuple

(29) 14. dimanche apokréô (dimanche VIII) (50v-52r)

Pour la même circonstance : nº4 (M II 124 ; J III 116), 108 (M II 124-125)

Renvoi pour l'abstinence de viande, le dimanche. Nous sommes comblés de tes biens Seigneur, par le saint mystère vivifiant

(30) $15.1^{\text {er }}$ dimanche de carême (dimanche VII) (52rv)

Pour la même circonstance : $\mathrm{n}^{\circ} 85$ (M II 125-126 ; J III 116-117)

$=\sin .54$, entre $\mathrm{n}^{\circ} 5$ et 6 ? (le microfilm omet les feuillets $33 \mathrm{v}-34 \mathrm{r}$ )

(53) D'après le microfilm; le catalogue accuse ici une faute de typographie.

(54) simdidrisa, litt. "richesses", double jeu de mots: avec "grande" (didi) et "tu t'es appauvris" (da-i-mcire).

(55) C'est le mot du ms. d'Adish (Lc 2, 28). 
Renvoi pour le premier dimanche des saints jeûnes. Espoir des extrémités de la terre ${ }^{56}$

(31) $16.2^{e}$ dimanche de carême (dimanche VI) (52v-53v)

Pour la même circonstance : $\mathrm{n}^{\circ} 86$ (M III 3 ; J III 117)

$=\sin .54$, entre $\mathrm{n}^{\circ} 5$ et 6 ? (le microfilm omet les feuillets $33 \mathrm{v}-34 \mathrm{r}$ )

Renvoi pour le deuxième dimanche. Nous nous livrons à toi, Seigneur notre Dieu et nous supplions la longanimité de ta miséricorde, toi qui connais les choses cachées

(32) 17. $3^{e}$ dimanche de carême (dimanche V) (53v-54r)

Pour la même circonstance : $\mathrm{n}^{\circ} 87$ (M III 4 ; J III 117-118)

$=\sin .54$, entre $\mathrm{n}^{\circ} 5$ et 6 ? (le microfilm omet les feuillets $33 \mathrm{v}-34 \mathrm{r}$ )

Renvoi pour le troisième dimanche. Vers toi qui as une abondance de miséricordes, ô Père de miséricordes, nous accourons (...). Fais-moi revenir vers toi, Seigneur, comme autrefois le fils devenu étranger à qui tu fais ${ }^{57}$ bon accueil.

(33) 18. $4^{e}$ dimanche de carême (dimanche IV) (54r-56r)

Pour la même circonstance : n88 (M III 4-5 ; J III 118 ; DM 10151016)

$=\sin .54, \mathrm{n}^{\circ} 6(34 \mathrm{v}-35 \mathrm{r})$

Renvoi pour le quatrième dimanche. Lorsque tu vis dans (ta) bonté le genre humain emmené par le démon (...) comme le pharisien (...) comme le publicain

(34) $19.5^{e}$ dimanche de carême (dimanche III) (56r-57v)

Pour la même circonstance : n89 (M III 5-6 ; J III 118-119)

$=\sin .54, \mathrm{n}^{\circ} 7(35 \mathrm{v}-36 \mathrm{r})$

Renvoi pour le cinquième dimanche. Nous levons les yeux de notre cœur et avec l'inclination de nos nuques [et] nous te demandons (de faire) miséricorde, ô très bon, toi qui as bien voulu envoyer la miséricorde des commandements (...) Lorsqu'un prêtre, Aaron, qui était premier grand-prêtre, le vit, il passa au loin ; un lévite qui descendait par ce chemin le vit, et il passa au loin

(35) 20. $6^{e}$ dimanche de carême (dimanche II) (58r-59r)

Pour la même circonstance : $\mathrm{n}^{\circ} 90$ (M III 6-7)

$=\sin .54, \mathrm{n}^{\circ} 8(36 \mathrm{v})$

(56) Suite de la traduction dans BBGG.

(57) Litt. vous faites. 
Renvoi pour le sixième dimanche. Toi qui par la Loi et les Prophètes enseignas l'espoir de la vie éternelle mais par les saints évangiles et les apôtres as révélé la béatitude ineffable, l’héritage de la Jérusalem céleste (...) Délivre-nous de la terrible géhenne, dans le feu inextinguible

\section{(36) 21. Commun des hiérarques (59r-60v)}

Pour la même circonstance : ${ }^{\circ} 154$ (J II 129 ; M IV 3)

$=\sin .54, \mathrm{n}^{\circ} 3(30 \mathrm{r}-31 \mathrm{v})$

Renvoi pour les saints hiérarques. Toi qui devins grand-prêtre pour nous selon l'ordre de Melchisédeq, non par la loi du commandement de la chair mais par la force de la vie inéluctable

(37) 22. Samedi de Lazare (60v-61r)

Pour la même circonstance : ${ }^{\circ} 92$ (M III 8 ; J III 131)

$=\sin .54, \mathrm{n}^{\circ} 9(37 \mathrm{v}-38 \mathrm{r})$

Renvoi pour la résurrection de Lazare. Toi qui as autorité sur la vie et la mort, ô Seigneur

(38) 23. Dimanche des Rameaux (61r-62v)

Pour la même circonstance : $\mathrm{n}^{\circ} 12$ (PK 27-28 ; J II 127-128) ; 93 (M III 8-9 ; J II 113-114 ; PK 21-22) ; 122 (M III 9-10 ; J III 119-120) ; 178 (Кo 95r-96r)

$=\sin .54, \mathrm{n}^{\circ} 10(38 \mathrm{r}-39 \mathrm{r})$

Renvoi pour les Rameaux. Les anges dans les cieux se prosternent devant toi, ô Seigneur, et sans attendre, que leur voix orne ton trône invisible! Mais nous de la terre

(39) 24. Grand jeudi (62v-63v)

Pour la même circonstance : ${ }^{\circ} 13$ (PK 107-108), 94 (M III 61-62), 95 (M III 63-64 ; J II 114 ; III 122-123), 117 (M III 62-63 ; J III 120-121)

$=\sin .54, \mathrm{n}^{\circ} 11(39 \mathrm{r}-40 \mathrm{r})$

Renvoi pour le grand jeudi. Ô roi éternel, Christ notre Dieu, toi qui as été éloigné du Père - et pour notre salut ton Verbe véritable a été envoyées ${ }^{58}$ sur la terre, de la Vierge par l'annonce d'un ange tu as fait resplendir dans le monde le soleil de justice -, toi le donateur de la Loi et celui qui l'accomplit, qui [as célébré] ton mystère sur le siège ${ }^{59}$ avec tes disciples lors de ton saint repas avec eux ...

(58) La terminaison du verbe est confuse, on lit un $a$ (3e pers. sg.) corrigé en $e$ (2e pers. sg.) ou inversement.

(59) Allusion au trône de Jacques conservé à Sion à l'époque byzantine, v. en dernier lieu Verhelst, 2003, p. 191 et IDEM, Liturgia, ch. 5, ad notes 260-261. 
(40) 25. grand samedi (63v-64r)

Pour la même circonstance : $n^{\circ} 14$ (PK 188-189 ; M III 65), 96 (M III 65), 118 (M III 66), 160 (BBGG)

$=\sin .54, \mathrm{n}^{\circ} 12(40 \mathrm{r})$

= M III 125-126; J I 28

Renvoi pour le Grand Samedi, le soir. Toi qui as brisé les portes d'airain et les barres de fer ${ }^{60}$

(41) 26. Pâques (64r-65v)

Pour la même circonstance : n 15 (PK 202-203 ; M III 121-123 ; J II 115 ; III 121), 97 (M III 124-125 ; J I 30 ; III 133), 119 (M III 123-124 ; J I 29)

$=\sin .54, \mathrm{n}^{\circ} 13(40 \mathrm{v}-41 \mathrm{v})$

Renvoi de l'assemblée (du jour) de l'accomplissement. Gloire à toi, ô Christ dispensateur de la lumière de connaissance ${ }^{61}$ qui luit (Jn 5, 35), trésor, agneau de Dieu qui enlèves les péchés du monde (Jn 1, 29), berger véritable qui donnes ta vie pour tes brebis (Jn 10,11), toi qui pensais dans la loi à la vie de ceux qui t'espèraient, tu t'es tu devant des juges qui ne connaissaient pas ton trône

(42) 27. Dimanche Nouveau (65v-66v)

Pour la même circonstance : ${ }^{\circ} 98$ (M III 126 ; J I 30), 126 (J I 30-31), 157 (M IV, 9 ; J III 121-122)

$=\sin .54, \mathrm{n}^{\circ} 14(41 \mathrm{v}-42 \mathrm{v})$

Renvoi pour le Dimanche Nouveau. Ô Dieu de lumière et source des biens, ton Fils-Unique et ton Esprit saint par lesquels tu as illuminé les créatures et tu as fait la vie au milieu des extrémités du monde, (...) car il est entré les portes closes chez les disciples

(43) 28. Ascension (66v-67v)

Pour la même circonstance : n¹00 (M III 129 ; J I 34 ; III 129-130), 127 (J I 33-34), 162 (J II 115-116)

$=\sin .54, \mathrm{n}^{\circ} 15(42 \mathrm{v}-43 \mathrm{v})$

Renvoi pour l'Ascension. Ô Dieu qui reçois la vénération de tous les saints, qui depuis le commencement dans la sainteté t'ont béni, et qui visites le lieu de ténèbre, pour lequel dans la désobéissance

(44) 29. Pentecôte (67v-68r)

Pour la même circonstance : n¹00 (M III 129 ; J I 34 ; III 129-130), 101 (M III 130-131 ; J I 35 ; III 130), 128 (J I 34-35 ; II 116 ; III 135-136 ; M III 131)

(60) Suite de la traduction dans BBGG.

(61) Litt. connue ; traduction de $\lambda$ 


$$
\begin{aligned}
& =\sin .54, \mathrm{n}^{\circ} 16(43 \mathrm{v}-44 \mathrm{r}) \\
& =\mathrm{n}^{\circ} 128
\end{aligned}
$$

Pour la Pentecôte. Écoute-nous, ô Seigneur notre sauveur, espoir de toutes les extrémités de la terre et [de] ceux qui sont au milieu de la mer en ces saints jours de la cinquantaine, toi qui as accordé à la race des hommes la réjouissance et la grâce et as répandu la venue de l'Esprit saint de façon visible sous l'apparence de langues de feu sur les disciples, et ils apparaitront comme évangélistes et ministres. Nous aussi tes serviteurs, conduis-nous par la grâce et la joie qui sont de toi

(45) 30. Nativité de Jean-Baptiste (68r-69r)

Pour la même circonstance : n¹49 (J II 129 ; II 133-135)

$=\sin .54, \mathrm{n}^{\circ} 19(46 \mathrm{r}-47 \mathrm{v})$

Pour la nativité de Jean. Ô Dieu sans commencement, Verbe du Père et coexistant au très-saint Esprit, toi qui par incommensurable amour-des-hommes es venu sur terre pour chercher les brebis perdues de la maison d'Israël (Mt. 15, 24) et qui as jugé acceptable d'habiter le sein d'une vierge

(46) 31. Athénagène, $7^{e}$ dimanche après la Pentecôte (69r-70v) $=\sin .54, \mathrm{n}^{\circ} 18(44 \mathrm{v}-46 \mathrm{r})$

Renvoi pour vardoba. Grand disciple et grand-prêtre, de même conseil, être, trône que ton père impérissable et sans commencement, Jésus, Verbe, Fils et Dieu avant les siècles, toi qui as désigné parmi tous les hommes tes saints apôtres pour servir dignement ta venue dans la chair

(47) 32. Transfiguration (70v-71v)

Pour la même circonstance : n¹04 (M III 186-187; J I 37-38), 131 (J I 36-37)

$$
=\sin .54, \mathrm{n}^{\circ} 20(47 \mathrm{v}-48 \mathrm{v})
$$

Pour la Transfiguration. Toi qui vois, tu es par divinité et indicible nature et existence immuable, image et éclat de la gloire du Père

(48) 33. Dormition (71v-72v)

Pour la même circonstance : n¹05 (M III 190-192; J I 38), 132 (J I 39 ; J III 124-125 ; M III 187), 159 (J III 127-128)

$=\sin .54, \mathrm{n}^{\circ} 21(48 \mathrm{v}-49 \mathrm{r})$

Pour mariamoba. Ô Dieu parfait et sans commencement, né de Dieu, Père, merveilleusement, Fils de même principat, et roi avec le Saint-Esprit, (...) tu n'as pas (été) corrumpu à la naissance mais as conservé ineffablement le mystère, semblablement aujourd'hui tu as transféré sans altération l'arche, ton repos (v. Ps. 131, 8) et le lieu, la 
tente (v. Ps. 131, 3) de ta gloire, dans les cieux sans destruction. Tu l'as conservée dans un royaume sans fin, par une connaissance plus haute que les pensées. Tu l'établis plus haut que toute créature pour être une lumière éclatante et qui ne passe pas, qu'aucun être humain n'a(vait jamais) vue, que tu réalises par une providence de ta divinité pour ceux qui errent

Remarque : Le Ps. 131, 8 est utilisé le 15 août dans la vieille version arménienne du lectionnaire de Jérusalem, avec station au Kathisme, alors que le canon du jour s'est modifié lors du déplacement de la station dans la fondation du roi Maurice (582-602) à Gethsémani (lectionnaire géorgien, $\mathrm{n}^{\circ} 1148$ ). Cette prière est donc antérieure au VII ${ }^{\mathrm{e}}$ siècle.

(49a) 34. Décollation de Jean-Baptiste, 29/08 (72v-74v)

Pour la même circonstance : $\mathrm{n}^{\circ} 102$ (M III 188; J I 39)

Pour la Décollation. Ô Dieu, toi qui as été prophétisé à l'origine et annoncé à l'avance par tous tes saints prophètes pour venir sur terre dans la chair et chercher ton peuple dispersé, ${ }^{62}$ qui a été rendu semblable à toi et a été détourné des vilains péchés

(49b) Le $\sin .54, \mathrm{n}^{\circ} 22(49 \mathrm{r}-50 \mathrm{v})$, contient une autre prière pour cette fête, dont le premier mot est Pirvel (premier, ou avant).

(50) 35. Nativité de la Théotokos (74v-76r)

Pour la même circonstance : $\mathrm{n}^{\circ} 71$ (M II 70-71), 105 (M III 190-192; J I 38), 180 (Ko 105v-106v)

$=\sin .54, \mathrm{n}^{\circ} 23(50 \mathrm{v}-52 \mathrm{r})$

Pour la nativité de la Théotokos. Rendus dignes d'atteindre la maison immaculée de ton héritage non corrompu, la génitrice-de-Dieu, mère et vierge, nous tes indignes serviteurs, t'adorons, ô Christ, et glorifions la Demeure de gloire, car

(51) 36. Encénies, 13/09 (76r-v)

Remarque : Outre le $\mathrm{n}^{\circ} 53$ ci-dessous, pour le 13 septembre, il existe une prière grecque pour toute dédicace d'église, le n¹24 (PAs 166-167).

(Renvoi) de l'Encénie. Toi, saint Seigneur et sanctificateur de tes saintes Églises, que tu as acquises par ton sang précieux, nous le rassemblement tout entier, en elles, des croyants, te glorifions, t'adorons, car

(52) 37. Exaltation, 14/09 (76v-77v)

Pour la même circonstance : $\mathrm{n}^{\circ} 11$ (J II 123-124) ; 53 (ci-dessous) ; 72 (M II 71-72) ; 181 (Ko 107r-108r)

(62) Sans doute $\pi \lambda \alpha \nu \omega ́ \mu \epsilon \nu o s$, errant 
Pour l'exaltation des croix. Bon berger qui as donné ta vie pour tes brebis (Jn 10,11), ${ }^{63}$ toi qui es arrivé aux portes de l'enfer par ta croix et as fait resplendir une lumière de vie pour ceux qui étaient assis dans la ténèbre et les ombres de la mort, et tu as mis fin $\grave{a}^{64} l^{\prime}$ errance de l'arbre et dépouillé l'enfer et mis à mort la mort et relevé Adam et fait sortir ceux qui étaient reclus dans les ténèbres, oui Seigneur, vraiment puissant, donne-nous ta croix précieuse comme protection et refuge des âmes et des corps. En elle établis les saintes églises et par elle accorde la victoire à nos rois, (et) par elle accorde la paix au monde.

Remarque : La demande pour les rois, dans cette prière, est l'indication d'une recension très tardive, peut-être du $\mathrm{X}^{\mathrm{e}}$ siècle..$^{65}$

(53) 38. Encénies et exaltation de la croix (77v-78v); Encénies $\left(\sin .54, n^{\circ} 24,52 r-53 r\right)^{66}$

Pour la même circonstance : v. ci-dessus $n^{\circ} 51$

$=\sin .54, \mathrm{n}^{\circ} 24(52 \mathrm{r}-53 \mathrm{r})$

Texte géorgien en appendice à cet article

Remarque 1 : Étant donné les difficultés de la traduction, j'ai exceptionnellement recensé le texte sur celui du sin. 54, qui présente une formulation parfois assez différente, que je reproduis à la suite de celle du sin. 12. Je traduis en mettant en évidence les difficultés du texte, qui est manifestement corrompu. Cela permet de voir que le texte du $\sin$. 54 semble meilleur (plus ancien) que celui du $\sin$. 12. Le copiste du $\sin .12$ a pu avoir devant les yeux le texte du $\sin$. 54 où il aurait ajouté la formulation complète des catégories d'anges empruntées à Col. 1, 16, dont il aurait modifié le " en lui » en un curieux « à côté de lui » (v. note 67), et qui semble avoir réagi contre l'idée du sin. 54, du commencement des anges dans (litt. par) la nature divine.

$\sin$. 12. Pour la dédicace des églises et l'exaltation des croix. Ô Dieu sans commencement, qui as engendré le Verbe sans commencement Dieu de commun conseil - par qui tout fut créé, sur-et-sous les cieux (Col. 1, 15), et tout ce qui est à côté67 de lui, trônes, dominations, principautés, pouvoirs et puissances (Col. 1, 16), classe ordonnée des an-

(63) Allusion à la lecture du jour dans LG : Jn 10, 22-37 / 42 (cf. Jn 10, 27).

(64) Litt. dissipé

(65) Voir BBGG.

(66) D'après le Catalogue (Gvaramia et al., p. 60, IV.1.24), la fin de la prière dit d'ajouter une partie " pour la Croix », qui est le texte $\mathrm{n}^{\circ} 17$ de ce manuscrit (ci-dessous n59).

(67) Zeda litt. sur, au-dessus 
ges, innombrable et lumineuse et sans fin, éternelle et invisible, à qui tu n'as pas montré le commencement des hommes ${ }^{68}$ dans (litt. selon) $l^{\prime}$ image et ressemblance de la nature ${ }^{69}$ divine. Et tu as rendu manifeste le ciel avec un luminaire et sa terre éternelle. Tu as déterminé tout commencement et fin avec providence avant (la venue de) ton fils actuel véritable. D'abord tu as révélé la terre hors des eaux comme d'une vierge, $\operatorname{par}^{70} l^{\prime}$ incarnation - et au milieu, le paradis de vie revêtu de forces, de l'édification de l'arbre de la croix.

sin. 54. Renvoi de l'assemblée des Encénies. Ô Dieu sans commencement, qui as engendré le Verbe sans commencement - Dieu de commun conseil - par qui toutes les choses elles-mêmes ont été créées sur-et-sous les cieux et ce qui est en lui, trônes, dominations et les classes ordonnées des anges invisibles, dont tu n'as pas montré le commencement aux hommes dans (litt. à cause de) la nature de la divinité. Et eux aussi (sont) avec un corps céleste ${ }^{71}$, et tu as rendu manifeste le commencement de la terre éternelle, et la fin de tous tu l'as déterminée avec providence avant ta venue actuelle véritable. D'abord tu as révélé la terre hors des eaux comme d'une vierge, par l'incarnation. Et au milieu du paradis, était annoncée la force de la vie revêtue, l'édification de la croix.

Remarque 2 : La suite commune aux deux manuscrits est une exégèse de la vision de Jacob dans Gn. 28, avec la fondation de la «maison de la divinité » (Béth-el).

Remarque 3 : On peut interpréter le texte dans deux sens divergents : Dieu n'a pas révélé aux anges qu'il allait créer les hommes, dont la nature est divine, à son image et sa ressemblance (ces derniers mots étant propres au $\sin .12)^{72}$; ou Dieu n'a pas révélé aux hommes le création des anges, dont la nature est divine. Ce dernier sens semble préférable d'après la suite où il est question de la nature angélique, éternelle et semblable à des corps célestes (?).

(68) Litt. par qui tu n'as pas montré le commencement aux hommes

(69) En corrigeant le nominatif en un génitif. Le copiste semble avoir compris les anges «par qui tu n’as pas manifesté aux hommes la nature de la divinité », mais les autres mots en deviennent («le commencement », «à l'image et à la ressemblance ») incompréhensibles.

(70) hors de (génitif)

(71) Ou «luminaire».

(72) On pourrait rappocher ce thème de celui de la jalousie des anges au moment de la création des hommes, v. par exemple Jean de Bolnisi, V 20. 
Remarque 4 : Dans le premier paragraphe de la prière, le $\sin .12$ dit explicitement des anges qu'ils sont éternels, comme dans la prière $\mathrm{n}^{\circ} 22$ («toutes les choses elles-mêmes de lui ont été faites, éternelles»); l'adjectif « sans commencement » dit de Dieu et du Verbe s'oppose au « commencement » dit des hommes. Les anges sont donc créés par le Verbe et éternels, donc, d'une certaine manière, éternellement créés. Ou encore : ils ont un commencement éternel. On notera aussi la création des anges en lui dans le sin. 54 (" tout ce qui est en lui »), avec la postposition šina, au lieu de zeda dans le sin. 12. On pressent à l'arrière-plan de ces difficultés de transmission textuelle des spéculations pro- ou anti-origénistes, ce que viendrait confirmer le curieux « corps céleste » (ou «luminaire»), qui semble bien s'appliquer aux anges dans le $\sin .54$, ainsi que la « terre éternelle » .

(54) 39. Zacharie, mutisme (78v-80r)

Pour le mutisme de Zacharie. (Ô toi qui) as saisi, une fois, la sainteté intouchable par le voile de ta chair, (toi qui étais) grand prêtre dans la maison de Dieu, sans commencement en même temps que le Père et Seigneur en même temps que le Saint-Esprit, Dieu véritable et homme fidèle, à la consommation des temps (tu as) pris chair de la sainte Vierge

(55) 40. Archanges (80r-v)

Pour la même circonstance : n74 (M II 73-74), 114 (M III 192 ; J III 127)

$=\sin .54, \mathrm{n}^{\circ} 25$

Pour les saints archanges. Dieu des esprits et de toute chair, (de $)^{73}$ tous les chevaliers célestes, trônes, dominations, pouvoirs et puissances, ceux qui se tiennent au-dessus, ${ }^{74}$ Séraphins aux six ailes et Chérubins aux yeux innombrables, ô toi qui fis tes anges en esprit et tes serviteurs en flamme de feu, bénis toutes les puissances lumineuses

(56) 41. Commun des martyrs (81r)

Pour la même circonstance : ${ }^{\circ} 23$ (ci-dessus), 110 (M III 194), 129 (J I 36), 130 (J I 36), 153 (M III 193-194), 169 (M III 194)

$=\sin .54, \mathrm{n}^{\circ} 26(53 \mathrm{rv})$

Pour les saints témoins. ${ }^{75}$ Dieu, fondateur du travail des saints et victorieux témoins, en bien

(73) Litt. dont

(74) Allusion aux vingt-quatre vieillards d'Ap. 4, 4 ?

(75) C'est-à-dire martyrs 
(57) 42. Commun des saints (81r-v)

$=\mathrm{n}^{\circ} 23$ (avec une variante dans l'incipit : Dieu des saints, qui etc.)

(58) 43. Quarante martyrs (81v-83r)

Pour la même circonstance : $\mathrm{n}^{\circ} 148$ (J II 126-127)

Pour les saints Quarante. Toi qui as rendu le témoignage d'une bonne confession devant Ponce Pilate

(59) 44. Croix (83r-v)

$=\sin .54, \mathrm{n}^{\circ} 17$ (44rv)

Remarque: La place de la prière dans le sin. 54, entre la Pentecôte $\left(n^{\circ} 44\right)$ et Athénagène $\left(n^{\circ} 46\right)$, montre qu'il s'agissait à l'origine d'une prière pour la fête de l'apparition de la Croix le 7 mai. ${ }^{76}$ Ce manuscrit fait en outre un renvoi au n ${ }^{\circ} 17$ à la fin du n ${ }^{\circ} 53$ (v. note 66).

Renvoi de la Croix. Béni es-tu Dieu d'Israël

(60) 45. Défunts (83v-84v)

Pour la même circonstance : $\mathrm{n}^{\circ} 116$ (M II 123)

Pour le salut de ceux qui se sont endormis. Toi qui as incliné les cieux et es descendu sur la terre

(61) 46. (1 $\left.{ }^{\text {er }}\right)$ dimanche $(84 v-85 r)$

Pour les dimanches ordinaires : $\mathrm{n}^{\circ} 9$ (CL ; M IV 3-4), 10 (CL ; M IV 3-4; J I 44-45; DM 43), 109 (M IV 11 ; J I 22-23), 112 (M IV 8-9 ; J I 44, 47), 113 (M IV 6-7 ; J I 42), 166 (M IV 4), 167 (M IV 5), 168 (M IV 6)

Renvois pour les saints dimanches. Roi des rois et Seigneur des Seigneurs ... qu'aucun humain n'a jamais vu

(62) $47.2^{e}$ dimanche $(85 r-v)$

$=\mathrm{n}^{\circ} 1$

$=\sin .54, \mathrm{n}^{\circ} 28(54 \mathrm{v})$

Pour un dimanche 2. Ceux que tu as appelés, ceux que tu as sanctifiés, Seigneur

(63) $48.3^{e}$ dimanche $(85 v-86 v)$

$=\sin .54, \mathrm{n}^{\circ} 29$ (55rv)

Pour un dimanche 3. Roi des rois et Seigneur des Seigneurs ... tu as trouvé ton peuple

(64a) 49. $4^{e}$ dimanche (86v-87v)

$=\sin .54, \mathrm{n}^{\circ} 27,1^{\mathrm{er}}$ dimanche $(54 \mathrm{rv})$

(76) PO 36, p. 333; CSCO, 204-205, nº57. 
$\approx \mathrm{n}^{\circ} 168^{77}$ (incipit, en italiques dans la traduction qui suit; v. aussi $\left.\mathrm{n}^{\circ} 66\right)$

Pour un dimanche 4. Gardien de notre vie, Seigneur Dieu qui seul es sans péché, Dieu source de vie et de bienfaits, et roi de tes créatures, prends en pitié ton peuple et garde ton héritage

Remarque: L'incipit de la prière du $4^{\mathrm{e}}$ dimanche dans le sin. 54, la dernière de la collection dans ce manuscrit, est omis dans le Catalogue. Traduction :

(64b) Renvoi de l'assemblée pour un $4^{e}$ dimanche. Seigneur de tous, souverain, Père de ton Christ, ceux qui ouvertement t'appellent, écoute-les ainsi que ceux qui en silence te vénèrent

(65) $50.5^{e}$ dimanche $(87 v-88 r)$

Pour un dimanche 5. Béni sois-tu, Seigneur en tout temps, toi qui nous gardes de tout mal et gardes l'entrée de nos oreilles et la sortie de nos lèvres

(66) 51. $6^{e}$ dimanche (88r-89r)

Pour un dimanche 6. Gardien de notre vie, Seigneur sans péché, toi qui par la bouche des prophètes nous annonçais ta venue sur la terre

(67) 52. Ordinaire (89r)

Pour la même circonstance : n`1-6 (v. ci-dessus), 109 (M IV 11; J I 22-23), 121 (M IV 10), 136 (J I 43), 157 (J III 121-122 ; M IV 9)

Renvois de toujours. Tu es glorifié, Créateur et Roi de tout, et nous adorons ton saint nom ineffable digne d'être servi, que tu as révélé à nous tes serviteurs

(68) 53. Ordinaire $b(89 r-v)$

De toujours 2. Seigneur notre espoir, asile, force, souverain, protecteur, tu es Dieu, nous te chantons

(69) 54. Ordinaire c (89v-90r)

De toujours 3. Seigneur tout-puissant, la source de tes bontés demeure toujours, profit des âmes, dans la grâce d'une bonne œuvre

(70) 55. Ordinaire d (90r)

De toujours 4. Nous te livrons nos âmes et de tout ton peuple à qui tu as répondu par le sang précieux de ton Christ

(77) Minisci, IV, p. 6. 


\section{Conclusion}

La présente étude ne porte pas sur l'ensemble des prières de renvoi géorgiennes, mais prend tout de même en compte tous les incipit (au minimum) des prières de la collection géorgienne des $\sin .12$ et 54. Sans revenir sur les conclusions de mon précédent article sur ce sujet, ${ }^{78}$ elle a permis d'avancer, me semble-t-il, deux nouveaux éléments de datation confirmant ma conclusion précédente, à savoir que la Vorlage grecque de la collection géorgienne remonte au VI ${ }^{\mathrm{e}}$ s. ${ }^{79}$ et même avant le milieu de ce siècle, et le tournant anti-origéniste de Justinien.

À propos de la Dormition d'abord ( $\left.{ }^{\circ} 48\right)$, la référence au Ps. 131 indique un prokiménon qui est antérieur à la Fondation du roi Maurice à la fin du $\mathrm{VI}^{\mathrm{e}}$ siècle.

D'autre part, et c'est ici la découverte la plus stimulante pour une recherche future, la prière pour les Encénies $n^{\circ} 53$ comporte des termes ( « corps célestes », « terre éternelle», anges «créés en lui ») qu'il est difficile d'expliquer sans allusion aux spéculations origénistes, ce qui nous renvoie bien au VI ${ }^{\mathrm{e}}$ siècle. La prière $\mathrm{n}^{\circ} 51$ apparaît alors comme une nouvelle formulation du $\mathrm{n}^{\circ} 53$, quand la prière ancienne, du fait d'une transmission chaotique, n'était plus comprise. De plus le fait que la prière pour $\mathrm{l}^{\prime}$ Exaltation, le 14 septembre $\left(\mathrm{n}^{\circ} 52\right)$, paraît très récente à cause de la demande pour les rois, renvoie à une époque où, comme dans la vieille version arménienne du lectionnaire, le 14 septembre n'a pas encore pris les traits d'une fête spécifique de l'Exaltation de la Croix. ${ }^{80}$

(78) Sur les cinquante-cinq prières de la collection géorgienne, trois prières ont un modèle grec identique ou pratiquement identique : $\mathrm{n}^{\circ} 30$, 40 et 44 . Deux prières (étudiées dans l'autre article) ont un modèle grec dont la forme conservée est assez éloignée du leur : n`16 et 19 . Dans un cas, la similitude se limite à l'incipit pour la même circonstance : $\mathrm{n}^{\circ} 23$.

(79) Les trois arguments que j'avançais sont : 1 . la place de l'Annonciation au début de l'année liturgique ; 2 . l'origénisme de la prière n`22;3. l'absence $\mathrm{du}$ thème du lavement des pieds dans la prière du Grand Jeudi. On pourrait y ajouter le fait que le sin. 54, qui semble avoir une structure (et un texte) plus ancienne que celle du $\sin .12$ (v. ci-dessus n॰53 rem. 1), ne comporte peutêtre que sept prières de carême (seul un examen du manuscrit lui-même pourrait le dire, v. nº 30 à 32) au lieu de huit dans le $\sin .12$ (v. le point 4 de mon argumentation dans $B B G G)$.

(80) PO 36, p. 363 : on vénère la relique de la Croix (comme le Vendredi saint) mais sans office propre (renvoi à l'office de la veille). 
Aucun des éléments rassemblés ici ne se trouve, à plus ample informé, dans les prières grecques de l'ambon qui ont été transmises jusqu'à nous, ce qui montre l'importance primordiale de la collection géorgienne dans l'origine et l'histoire de ce genre euchologique.

\section{Appendice. Texte géorgien du $\mathrm{n}^{\circ} 53$}

$\sin .12,77 \mathrm{v}-78 \mathrm{v}$

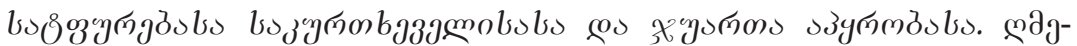

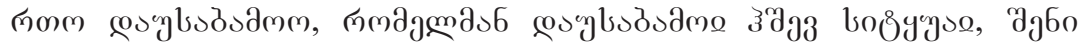

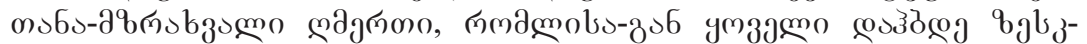

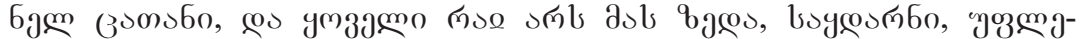

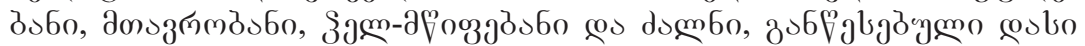

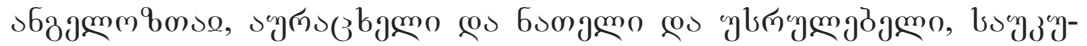

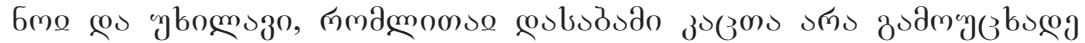

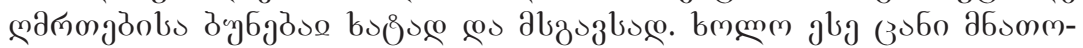

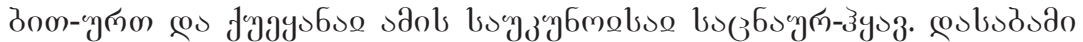

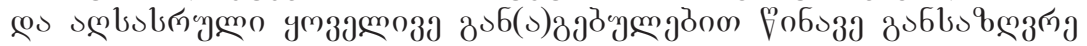

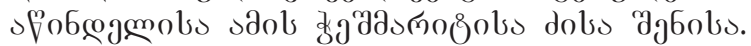

$\sin .54,52 \mathrm{r}-53 \mathrm{r}$

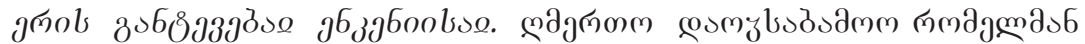

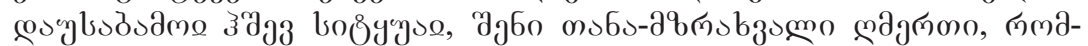

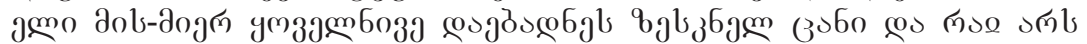

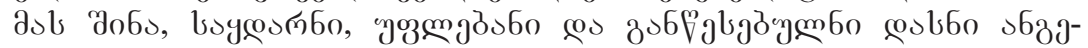

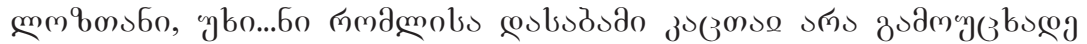

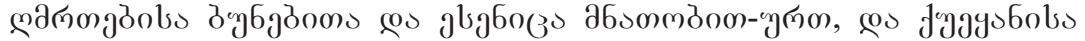

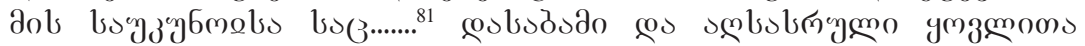

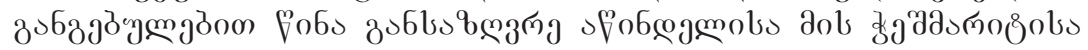
dmlsmalso gj6ols.

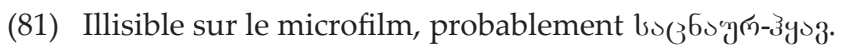

Bibliographie supplémentaire : A. Renoux, Le codex arménien Jérusalem 121, t. II. Édition comparée du texte et de deux autres manuscripts, introduction, textes, traduction et notes, dans PO 36 (1971), 141-390 ; M. TARCHNIšviLI, Le grand lectionnaire de l'Eglise de Jérusalem (Ve-VIII siècle), I et II (Louvain, 1959 et 1960) (CSCO, 188-189 et 204-205) ; S. Verhelst et al., Liturgia ibero-graeca sancti Iacobi Dei fratris. Georgian Text edited and translated under the Autority of the Institute of Manuscripts in Tbilisi. Rétroversion grecque et commentaire, à paraître dans la collection Jerusalemer Theologisches Forum (Münster). 


\section{RÉSUMÉ}

Cet article retrace la genèse de la prière de l'ambon dans la Liturgie de saint Jacques et établit ensuite la liste de ses variantes en grec et surtout en géorgien. Il comporte la traduction des incipit de 55 prières géorgiennes, faite directement sur le microfilm du sinaiticus 12 . L'analyse plus rapprochée de l'une d'entre elles ( $\left.{ }^{\circ} 53\right)$ apporte une nouvelle observation (par rapport à une première partie de cet article parue dans la revue $B B G G$ ) sur l'origénisme qui a marqué la tradition liturgique de Jérusalem avant Justinien. On y trouvera d'autres éléments de datation tendant à faire remonter la Vorlage grecque de la collection géorgienne (du moins de sa partie principale) avant le milieu du $\mathrm{VI}^{\mathrm{e}}$ siècle. 